\title{
Contact mechanics for poroelastic, fluid- filled media, with application to cartilage
}

Cite as: J. Chem. Phys. 145, 234703 (2016); https://doi.org/10.1063/1.4972067

Submitted: 30 September 2016 . Accepted: 29 November 2016 . Published Online: 19 December 2016

B. N. J. Persson
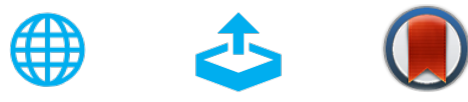

View Online

Export Citation

CrossMark

\section{ARTICLES YOU MAY BE INTERESTED IN}

Soft matter dynamics: Accelerated fluid squeeze-out during slip

The Journal of Chemical Physics 144, 124903 (2016); https://doi.org/10.1063/1.4944384

Theory of rubber friction and contact mechanics

The Journal of Chemical Physics 115, 3840 (2001); https://doi.org/10.1063/1.1388626

Multiscale physics of rubber-ice friction

The Journal of Chemical Physics 145, 114703 (2016); https://doi.org/10.1063/1.4962576

PHYSICS TODAY

WHITEPAPERS
ADVANCED LIGHT CURE ADHESIVES

Take a closer look at what these

environmentally friendly adhesive

systems can do
READ NOW

PRESENTED BY 8. MASTERBOND* 


\title{
Contact mechanics for poroelastic, fluid-filled media, with application to cartilage
}

\author{
B. N. J. Persson ${ }^{a)}$ \\ PGI-1, FZ Jülich, Germany
}

(Received 30 September 2016; accepted 29 November 2016; published online 19 December 2016)

\begin{abstract}
I study a simple contact mechanics model for a poroelastic, fluid-filled solid squeezed against a rigid, randomly rough substrate. I study how the fluid is squeezed out from the interface, and how the area of contact, and the average interfacial separation, change with time. I present numerical results relevant for a human cartilage. I show that for a fluid filled poroelastic solid the probability of cavitation (and the related wear as the cavities implode), and dynamical scraping (defined below and in Hutt and Persson, J. Chem. Phys. 144, 124903 (2016)), may be suppressed by fluid flow from the poroelastic solid into the (roughness induced) interfacial gap between the solids. Published by AIP Publishing. [http://dx.doi.org/10.1063/1.4972067]
\end{abstract}

\section{INTRODUCTION}

Tribology, the study of the interaction between solids in a stationary or sliding contact, ${ }^{1-4}$ is a topic of great importance in nature and in technology. In particular the tribology of soft matter, such as rubbers or gels, has drawn increasing attention, but is a very complex topic. ${ }^{5-13}$ One important class of soft tribology problems refers to the tribology of biological matter (i.e., material of living bodies). Thus, human body possesses exquisite systems of lubrication that can withstand thousands of work cycles per day without fatigue. ${ }^{8}$ The lubrication of large articulating joints or the eyelid-cornea interface is critical to the health and function of their constituent tissues. The articular cartilage of the knee (see Fig. 1(a)) is composed of a highly structured network of collagen fibers, hyaluronin, and lubricin produced by encased chondrocyte cells. Epithelial cells in the eye produce a protective and lubricating polymer network, the glycocalyx, which is composed of glycoprotein mucins, proteoglycans, and glycolipids. It is believed that the weeping lubrication at the surfaces of these nanoporous biopolymer networks is an important mechanism for the low friction and wear observed for these systems.

Here I study the contact between a porous fluid-filled elastic solid (see Fig. 2) with a flat surface, and a rigid solid with a surface having roughness on different length scales (see Fig. 1(c)). I use the so-called biphasic theory ${ }^{13,14}$ to describe the fluid filled porous solid. This theory assumes that the solid is a micro (or nano) porous material with fluid filled and interconnected cavities and may be a useful model for the articular cartilage or the smooth adhesive pads of some insects (e.g., crickets) and tree frogs. ${ }^{15,16}$

The constitutive equations in the biphasic model of the tissue are equations for the solid (apparent) stress, the fluid (apparent) stress, and a diffusive resistance of relative motion depending on the (local) relative solid and fluid velocities.

a)URL: www.MultiscaleConsulting.com.
Under the action of compressive loading, the deformation of the solid matrix and the flow of the interstitial fluid give rise to the relative motion between the two phases. This model has many applications and has been used to describe the articular cartilage. ${ }^{13,14}$ However, no one has so far been able to solve the equations in a realistic situation where two solids with surface roughness on many length scales are squeezed into contact. To study this problem, I combine the biphasic theory with the Reynolds equation of fluid flow between solid walls ${ }^{17}$ and the Persson contact mechanics theory for randomly rough surfaces. ${ }^{18,19}$ Using this theory, one can study processes like the one illustrated in Fig. 3. In this paper, I consider only the limiting case where the fluid in the porous solid flows relative to the elastic network orthogonal to the nominal contact area.

The fluid squeeze-out from a poroelastic solid has been studied in the past both theoretically and experimentally by Mow and co-workers. ${ }^{13,14}$ In these studies, a free-draining, rigid filter was squeezed against a cylindrical plug of the cartilage in a rigid confining chamber (see Fig. 1(b)). The load or squeezing force was constant and the vertical displacement of the filter was measured as a function of the squeezing time. Good agreement was found between the theory and the experimental data for the human cartilage. Note that the fluid flow channels in the rigid filter were so large that the resistance towards the fluid squeeze-out from the filter was negligible. Thus the fluid pressure in the filter was nearly equal to the external (atmospheric) pressure.

Here we consider a compact solid squeezed against the poroelastic solid, and in this case the fluid is removed via the open channels which occur at the interface as a result of the surface roughness (see Fig. 1(c)). However, these channels are in general very narrow and a large resistance towards fluid squeeze-out results from the viscous drag on the fluid as it moves in the narrow interfacial channels. As a result, the fluid at the interface will be pressurized and will carry a part of the external load. We will describe the fluid flow at the interface using the Reynolds equation with a source term 


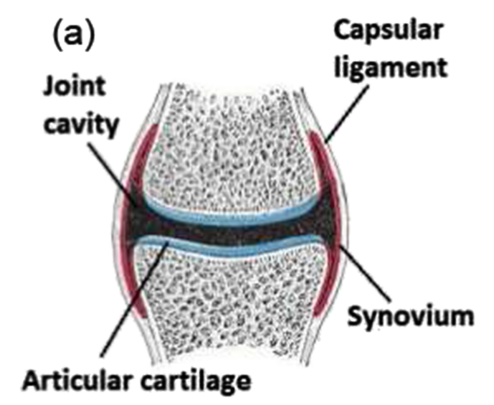

(b)

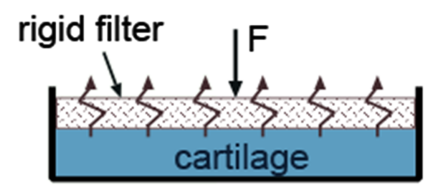

(c)

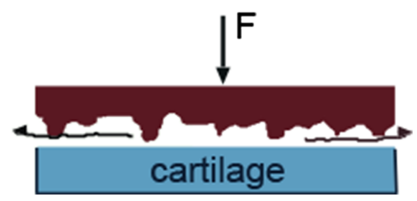

FIG. 1. (a) An animal joint. (b) A rigid porous membrane (filter) squeezed against a cartilage. The pore channels in the filter are large enough so that there is negligible resistance to fluid flow in the filter. In Refs. 13 and 14, the fluid squeeze-out from the cartilage was studies as a function of time and was used to determine the cartilage effective elastic modulus and fluid diffusive resistance, and to test the applicability of the biphasic theory for the cartilage. (c) Fluid squeeze-out studied in this paper. A rigid compact solid with a randomly rough surface is squeezed against a poroelastic solid. The fluid flow at the interface between the solids in a complex system of narrow channels.

derived from the fluid squeeze-out from the poroelastic solid. Although we focus on the case of a poroelastic solid with a flat surface squeezed against a rigid randomly rough countersurface, the results are also valid for one rough poroelastic solid squeezed against another rough poroelastic solid. In this case, the relevant roughness is the combined roughness of the two surfaces. In addition the fluid will enter (or leave) the interfacial region from both of the poroelastic solids, i.e., the source term in the Reynolds equation will have contributions from both walls.

We emphasize that the multiscale nature of roughness has a crucial influence on the fluid flow at interfaces. This is most easily seen as follows: ${ }^{20,21}$ Consider first the elastic contact between two solids with random roughness on many length scales. We study the interface between the solids with increasing magnification. At low magnification (say the naked eye), we do not observe any surface roughness and it appears as if complete contact occurs between the solids, i.e., $A=A_{0}$, where $A_{0}$ is the nominal contact area. We now increase the magnification and first observe only the long wavelength roughness which will deform elastically under the applied load so that $A$ is only slightly smaller that $A_{0}$. In this case, the contact area percolates, i.e., there is no open channel at the interface through which the fluid could flow from one side to the other side. As we increase the magnification further we observe shorter wavelength roughness and the (apparent) contact area $A$ decreases.

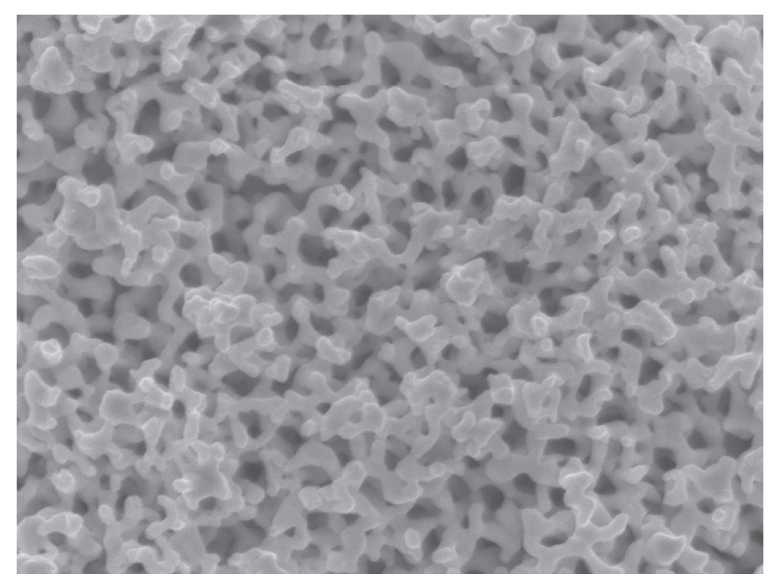

FIG. 2. Porous, fluid filled elastic material.
Exact numerical studies have shown ${ }^{22}$ that when the relative contact area reach $A / A_{0} \approx 0.42$ the non-contact area percolate and at this point the first open non-contact channel will appear, which allow fluid flow at the interface from one side to the other side (and would result, e.g., in the leakage of a seal). As the load (or nominal contact pressure) increases, the magnification where the non-contact area percolates will increase. Thus, depending on the external load different parts of the surface roughness spectrum will be important for the fluid flow. The discussion above does not include the influence of the fluid pressure on the contact mechanics (in the full theory it is included in a mean-field approach) but nevertheless shows that the multiscale roughness is of crucial importance for fluid flow at interfaces.

The fluid squeeze-out between elastic solids with surface roughness is a very complex topic. Here we use a theory which combines an analytical contact mechanics approach (see Refs. 18 and 19), with the Bruggeman effective medium theory $^{23}$ and the Reynolds equation ${ }^{24}$ for the fluid flow. For elastic solids, this theory was tested in Ref. 22 where we compared the fluid flow obtained using the analytical approach with the exact numerical studies. Other aspects of the theory were tested experimentally in Refs. 25 and 26 (fluid squeeze-out) and Refs. 20 and 21 (leak-rate of seals).

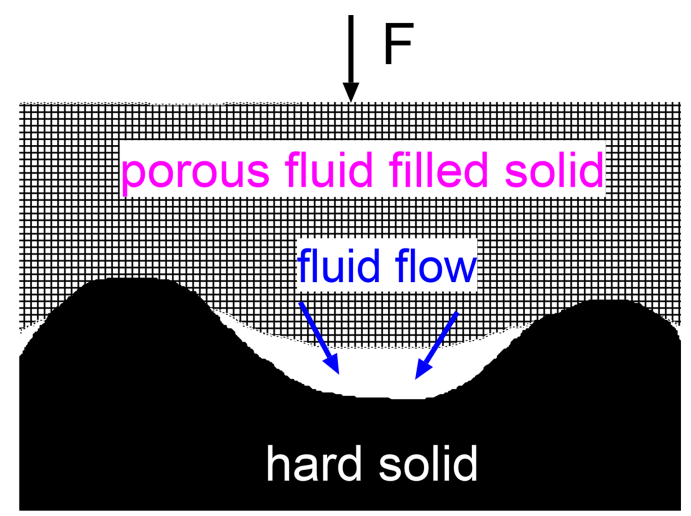

FIG. 3. The pressure in the asperity contact region generates a fluid pressure gradient in the porous material which forces the fluid to flow into empty or low-pressure fluid filled regions at the interface. The fluid-filled regions will carry a part of the external load and also acts as a lubricant at the onset of slip. 


\section{THEORY}

\section{A. Simple model of poroelastic solid}

In this section, I consider the simplest model possible of a porous elastic solid filled by a fluid, which is assumed to percolate in the solid. Consider an elastic solid with a regular array of cube-shaped cavities filled by a fluid, see Fig. 4(a). We assume that small circular holes or pores occur in the solid walls between the cavities. Thus when the solid deforms, fluid can move between the different cavities, but in this process the energy is dissipated into heat due to the viscosity of the fluid. We assume the walls have the thickness $d$ and the surface area of a side of the cubic cavity is denoted by $A_{1}$. The "lattice constant" is denoted by $B$, and we define $A_{0}=B^{2}$ and $A_{1}=(B-d)^{2}$. We consider a uniform compression in the normal direction (z-direction in Fig. 4(b)), and we assume that the vertical elastic walls deform (compression or elongation in the $z$-direction) without bending. We also assume that the thickness of the elastic sheets does not change during the compression, which corresponds to a Poisson ratio $v=0$ for the elastic solid.

If the material in the elastic walls have the Young's elastic modulus $E$, we can define an effective modulus as follows. If we compress a unit $B \times B$ by distance $u$ so the strain $\epsilon=u / B$ then the force to make the deformation will be $F=E(u / B)\left(A_{0}-A_{1}\right)$. We now define $E^{*}$ so that $F=E^{*}(u / B) A_{0}$ or $E^{*}=E\left(1-A_{1} / A_{0}\right)$.

Under the assumptions given above, the fluid volume conservation gives

$$
A_{1}\left(\dot{u}_{n+1}-\dot{u}_{n}\right)=\kappa\left(p_{n+1}-p_{n}\right)+\kappa\left(p_{n-1}-p_{n}\right)
$$

where $\dot{u}=d u / d t$ is the velocity of a node point as indicated in Fig. 4(b). We have assumed that the flow of fluid from the cavity $n+1$ to the cavity $n$ is proportional to the fluid pressure difference $\left(p_{n+1}-p_{n}\right)$ between the two cavities. The parameter $\kappa$ can be estimated by assuming the Poiseuille flow through $N_{0}$ (in the figure $N_{0}=1$ ) circular pores of radius $r_{0}$ much smaller than the thickness of the wall, ${ }^{27} \kappa \approx N_{0}(\pi / 8) r_{0}^{4} /(\eta d)$, where $\eta$ is the fluid viscosity and $d$ the thickness of the walls.

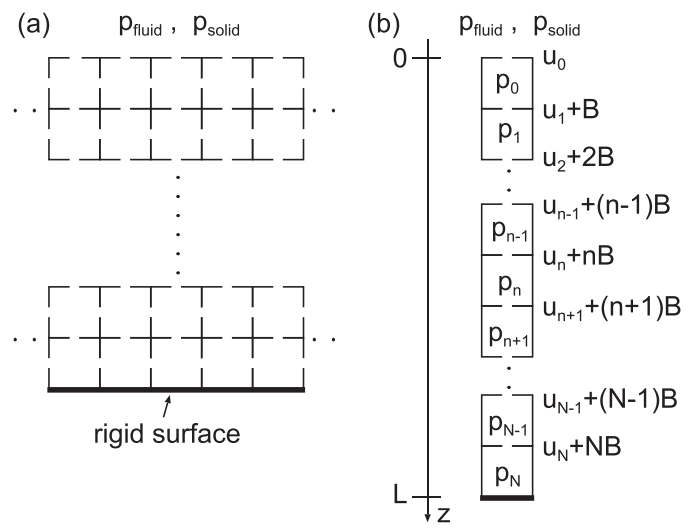

FIG. 4. (a) A simple model of a poroelastic solid. The material consists of a regular array of cubic cavities filled with a fluid and surrounded by elastic walls. The walls have small pores through which the fluid can flow. We consider a case where the solid is uniformly compressed in the normal $z$-direction and we assume that no side leakage of the fluid occurs. In this case, the problem in (a) can be mapped on the 1D problem in (b).
Neglecting inertia effects, the force balance gives

$$
A_{1}\left(p_{n}-p_{n-1}\right)+k\left(u_{n}-u_{n-1}\right)+k\left(u_{n}-u_{n+1}\right)=0,
$$

where $k \approx E d$, where $d$ is the thickness of the wall (or plate). Assume now that $u_{n}$ and $p_{n}$ change slowly with $n$ and let us denote $z=n B$. We consider $p_{n}$ as a function of $z$ denoted by $p(z)$. Expanding

$$
p_{n+1} \approx p_{n}+\frac{\partial p}{\partial n}=p(z)+B \frac{\partial p}{\partial z}(z)
$$

and similarly for $u_{n}$ we get from (1),

$$
A_{1} B \frac{\partial^{2} u}{\partial z \partial t}=\kappa B^{2} \frac{\partial^{2} p}{\partial z^{2}}
$$

and from (2),

$$
A_{1} B \frac{\partial p}{\partial z}=k B^{2} \frac{\partial^{2} u}{\partial z^{2}} .
$$

We can write (3) as

$$
c \frac{\partial^{2} u}{\partial z \partial t}=\frac{\partial^{2} p}{\partial z^{2}},
$$

where $c=A_{1} /(\kappa B)=(8 / \pi)\left(\eta / N_{0}\right)(d / B)\left(A_{1} / r_{0}^{4}\right)$. Integrating this equation with respect to $z$ gives

$$
c \frac{\partial u}{\partial t}=\frac{\partial p}{\partial z}+f(t)
$$

where $f(t)$ is a function of time only. We will show below that for $z=L$ both $u$ and $\partial p / \partial z$ vanish, and it follows that $f(t)=0$. Thus

$$
c \frac{\partial u}{\partial t}=\frac{\partial p}{\partial z} .
$$

From (4), we get

$$
\frac{\partial p}{\partial z}=K \frac{\partial^{2} u}{\partial z^{2}},
$$

where $K=k B / A_{1}$. Using that $k=E d$, we can also write $K=E B d / A_{1}=\left[E^{*} /\left(1-A_{1} / A_{0}\right)\right]\left(B d / A_{1}\right)=\mu E^{*}$, where $\mu$ is a geometrical factor. Combining (5) and (6) gives

$$
\frac{\partial u}{\partial t}=D \frac{\partial^{2} u}{\partial z^{2}},
$$

which is the standard diffusion equation with the diffusivity $D=K / c$.

To solve the equations above, we need boundary conditions on the surfaces $z=0$ and $z=L$. For $z=L=(N+1) B$, we have $u(L)=0$ and the volume conservation condition becomes

$$
A_{1}\left(\dot{u}_{N}-\dot{u}_{N-1}\right)=\kappa\left(p_{N-1}-p_{N}\right)
$$

or

or

$$
A_{1} B \frac{\partial^{2} u}{\partial z \partial t}=-\kappa B \frac{\partial p}{\partial z}
$$

$$
B c \frac{\partial^{2} u}{\partial z \partial t}(L)=-\frac{\partial p}{\partial z}(L) .
$$

As $B \rightarrow 0$, this condition reduces to

$$
\frac{\partial p}{\partial z}(L)=0 .
$$

Let us now consider the boundary condition at $z=0$. When applied to $n=1$ the volume conservation takes the form

$$
A_{1}\left(\dot{u}_{1}-\dot{u}_{0}\right)=\kappa\left(p_{1}-p_{0}\right)+\kappa\left(p_{\text {fluid }}-p_{0}\right),
$$


giving for $z=0$,

$$
A_{1} B \frac{\partial^{2} u}{\partial z \partial t}=\kappa B \frac{\partial p}{\partial z}+\kappa\left(p_{\text {fluid }}-p\right)
$$

or

$$
c B^{2} \frac{\partial^{2} u}{\partial z \partial t}=B \frac{\partial p}{\partial z}+\left(p_{\text {fluid }}-p\right) .
$$

In the limit $B \rightarrow 0$, this condition reduces to

$$
p(0)=p_{\text {fluid }} \text {. }
$$

Let us now study the force balance condition. We assume that the force $F$ act on the elastic solid at $z=0$. Thus the nominal solid contact pressure $p_{\text {solid }}=F / A_{0}$. In this case, (2) takes the form

$$
A_{1}\left(p_{0}-p_{\text {fluid }}\right)-k\left(u_{1}-u_{0}\right)-F=0
$$

or for $z=0$

$$
A_{1}\left(p-p_{\text {fluid }}\right)-k B \frac{\partial u}{\partial z}-F=0 .
$$

Since $p(0)=p_{\text {fluid }}$, we obtain

$$
-k B \frac{\partial u}{\partial z}=F .
$$

Dividing with $A_{0}=B^{2}$ gives

$$
-K \frac{A_{1}}{A_{0}} \frac{\partial u}{\partial z}(0)=p_{\text {solid }}
$$

In the equations above, $p$ is the pressure in the fluid but it is often more convenient to use the nominal fluid pressure $p^{*}$ defined so that $p^{*} A_{0}=p A_{1}$. In this case, $K$ in (6) is replaced by $E^{*}\left(1-A_{1} / A_{0}\right)(d / B)=E^{*}\left(A_{0}-A_{1}\right) d / V_{0}$, where $V_{0}=B^{3}$.

The boundary conditions derived above have a very simple interpretation within the continuum mechanical approach. Thus, $u(L)=0$ is trivial because at $z=L$ the bottom surface of the poroelastic solid is attached to a rigid wall. The condition $\partial p / \partial z=0$ for $z=L$ follows from the fact that the fluid current $J_{\perp}(z)$ is proportional to the gradient of the pressure (Darcy's law), $\partial p / \partial z$, and since $J_{\perp}(L)=0$, it follows that $\partial p / \partial z=0$ for $z=L$.

Consider now the boundary conditions for $z=0$. If the fluid pressure varies continuously in the vicinity of the surface $z=0$, then $p(0)=p_{\text {fluid }}$. Finally, the pressure $p_{\text {solid }}$ acting on the elastic solid is given by the normal components of the stress tensor which is proportional to $\partial u / \partial z$ in the present case. This gives the last boundary condition.

It is possible to define solid and fluid velocities. The solid velocity $v_{\text {solid }}=\partial u / \partial t$. The fluid velocity can be obtained by the following argument. Relative to the solid walls of cell $n$ the fluid move with the (average) velocity

$$
\frac{1}{A_{1}} \kappa\left(p_{n}-p_{n+1}\right) \approx-\frac{\kappa B}{A_{1}} \frac{\partial p}{\partial z} .
$$

The solid wall move relative to a fixed external coordinate system with the velocity $\partial u / \partial t$. Hence the fluid velocity

$$
v_{\text {fluid }}=\frac{\partial u}{\partial t}-\frac{\kappa B}{A_{1}} \frac{\partial p}{\partial z} \text {. }
$$

Since $\kappa B / A_{1}=1 / c$, we see from (5) that in the present case the fluid velocity vanishes. In Appendix A, we show how the equations derived above can be generalized to obtain the standard equations of poroelastic solids.

\section{B. Solution of the basic equations for arbitrary (time-dependent) fluid and solid contact pressures}

Let us define the Fourier transform

$$
u(\omega)=\frac{1}{2 \pi} \int d t u(t) e^{i \omega t}
$$

and similarly for $p(\omega)$. After the Fourier transformation of the time-dependency (5) and (7) gives

$$
\begin{gathered}
-i \omega c u=\frac{\partial p}{\partial z}, \\
-i \omega u=D \frac{\partial^{2} u}{\partial z^{2}} .
\end{gathered}
$$

If we introduce

$$
\gamma=\left(-\frac{i \omega}{D}\right)^{1 / 2}=(1-i)\left(\frac{\omega}{2 D}\right)^{1 / 2},
$$

then we can write the solution to (13) as

$$
u=u_{1}(\omega) e^{\gamma z}+u_{2}(\omega) e^{-\gamma z} .
$$

The boundary condition $u=0$ for $z=L$ gives

$$
u_{2}=-u_{1} e^{2 \gamma L},
$$

so that

Using (12), we get

$$
u=u_{1}(\omega)\left(e^{\gamma z}-e^{\gamma(2 L-z)}\right) .
$$

$$
p=-\frac{i \omega u_{1} c}{\gamma}\left(e^{\gamma z}+e^{\gamma(2 L-z)}\right)+g(\omega) .
$$

Note that the boundary condition $\partial p / \partial z=0$ for $z=L$ is obeyed.

The boundary condition (9) gives

$$
u_{1}=-\frac{A_{0}}{K A_{1}} \frac{p_{\text {solid }}}{\gamma\left(1+e^{2 \gamma L}\right)},
$$

and hence

$$
u(z, \omega)=-\frac{A_{0}}{A_{1}} \frac{p_{\text {solid }}}{K \gamma} \frac{e^{\gamma z}-e^{\gamma(2 L-z)}}{1+e^{2 \gamma L}} .
$$

Next, the boundary condition $p=p_{\text {fluid }}(\omega)$ for $z=0$ gives

$$
g=p_{\text {fluid }}-\frac{A_{0}}{A_{1}} p_{\text {solid }} \text {. }
$$

Thus

$$
p=p_{\text {fluid }}+\frac{A_{0}}{A_{1}} p_{\text {solid }}\left(1-\frac{e^{\gamma z}+e^{\gamma(2 L-z)}}{1+e^{2 \gamma L}}\right) .
$$

The flow of fluid from the poroelastic solid to the outside is given by the flow current

$$
J_{\perp}=\kappa \frac{B}{A_{0}} \frac{\partial p}{\partial z}=-\frac{B}{A_{1}} p_{\text {solid }} \kappa \gamma \frac{1-e^{\gamma 2 L}}{1+e^{2 \gamma L}} .
$$

Here we are interested in the fluid squeeze-out between a poroelastic solid and a rigid solid with a randomly rough surface. When the poroelastic solid is squeezed against another solid, the fluid current $J_{\perp}(\mathbf{x}, t)$ is a source field in the effective Reynolds equations for the fluid flow. That is, it enters in the continuity equation

$$
\frac{\partial u}{\partial t}+\nabla \cdot \mathbf{J}=J_{\perp}
$$

Here $u(\mathbf{x}, t)$ is the interfacial separation and $\mathbf{J}(\mathbf{x}, t)$ the $2 \mathrm{D}$ flow current parallel to the interface, where $\mathbf{x}=(x, y)$ denote the lateral position vector at the interface.

Let us now consider some limiting cases. 


\section{Time-dependent fluid pressure $p_{\text {fluid }}(t)$ outside the poroelastic solid}

In this case $p_{\text {solid }}=0$, and from (16) it follows that the fluid pressure $p(z, t)=p_{\text {fluid }}(t)$ is the same everywhere. This is of course the expected result for an incompressible fluid.

\section{Oscillating (in time) solid contact pressure while vanishing fluid pressure outside the solid}

Assume $p_{\text {solid }}(t)=p_{0}+p_{1} \cos \left(\omega_{0} t\right)$. In this case,

$$
p_{\text {solid }}(\omega)=p_{0} \delta(\omega)+p_{1} \frac{1}{2}\left(\delta\left(\omega-\omega_{0}\right)+\delta\left(\omega+\omega_{0}\right)\right),
$$

and from (15),

$$
\begin{gathered}
u(z, t)=\frac{A_{0}}{A_{1}} \frac{p_{0}}{K}(L-z), \\
-\frac{A_{0}}{A_{1}} \frac{p_{1}}{K} \operatorname{Re}\left[\frac{e^{\gamma_{0} z}-e^{\gamma_{0}(2 L-z)}}{\gamma_{0}\left(1+e^{2 \gamma_{0} L}\right)} e^{i \omega_{0} t}\right],
\end{gathered}
$$

where

$$
\gamma_{0}=\left(-\frac{i \omega_{0}}{D}\right)^{1 / 2} .
$$

The first term in (19) is just the static elastic deformation in response to the constant pressure $p_{0}$. We get

The fluid pressure is obtained from (16) with $p_{\text {fluid }}(t)=0$.

$$
p=\frac{A_{0}}{A_{1}} p_{1} \operatorname{Re}\left[\left(1-\frac{e^{\gamma_{0} z}+e^{\gamma_{0}(2 L-z)}}{1+e^{2 \gamma_{0} L}}\right) e^{i \omega_{0} t}\right] .
$$

Let us consider the limiting case of $\omega_{0}<<D / L^{2}$. In this case,

$$
u(z, t)=\frac{A_{0}}{A_{1}} \frac{1}{K}(L-z)\left[p_{0}+p_{1} \cos \left(\omega_{0} t\right)\right]
$$

and

$$
p(z, t)=-\frac{A_{0}}{A_{1}} \frac{p_{1}}{2 D} z(2 L-z) \omega_{0} \sin \left(\omega_{0} t\right) .
$$

Note that the solid deformation velocity field

$$
v(z, t)=\frac{d u}{d t}(z, t)=-\frac{A_{0}}{A_{1}} \frac{p_{1}}{K}(L-z) \omega_{0} \sin \left(\omega_{0} t\right) .
$$

Thus, the fluid pressure has the same time dependency as the solid velocity, which is expected as the fluid is dragged by the frictional coupling to the solid walls. Note also that $u(z, t)$ vanishes for $z=L$ as expected and takes its largest value at the surface $z=0$. The fluid pressure instead takes its largest value at the wall $z=L$ as expected because the drag force from the solid walls add up monotonically as we move from the outer surface $z=0$ (where the fluid pressure is assumed to vanish) to the wall $z=L$.

If we define the velocity

$$
v_{0}=\frac{A_{0}}{A_{1}} \frac{p_{1} L \omega_{0}}{2 K},
$$

we can write

$$
v(z, t)=-v_{0} 2\left(1-\frac{z}{L}\right) \sin \left(\omega_{0} t\right)
$$

and

$$
p(z, t)=-v_{0} L c \frac{z}{L}\left(2-\frac{z}{L}\right) \sin \left(\omega_{0} t\right) .
$$

Note that $p(L, t)=-c L v_{0} \sin \left(\omega_{0} t\right)$. This equation can be obtained directly from (5) by integrating $z$ from 0 to $L$,

$$
c \int_{0}^{L} d z v=p(L, t)
$$

or

$$
p(L, t)=c L\langle v\rangle,
$$

where $\langle v\rangle$ is the average velocity which according to (20) is $-v_{0} \sin \left(\omega_{0} t\right)$ in the present case. In Appendixes B and C, we consider the problem discussed above when $p_{\text {solid }}(t)$ has arbitrary dependency on time.

\section{CONTACT MECHANICS FOR RANDOMLY ROUGH SURFACES}

Here we consider the contact between a poroelastic solid with a nominal flat surface and a rigid solid with random roughness. The poroelastic solid is assumed to have the thickness $L$. We will consider the limit where the time-variation is so slow that $\omega<<D / L^{2}$, corresponding to long squeeze-out times. In this case, (17) reduces to

$$
J_{\perp}(\omega)=-\frac{L}{K} i \omega p_{\text {solid }}(\omega)
$$

or

$$
J_{\perp}(t)=\frac{L}{K} \frac{d p_{\text {solid }}}{d t}(t) .
$$

Using (15), we can write the (low frequency) elastic strain

$$
\epsilon=\frac{u(0, t)}{L}=\frac{1}{E} p(t),
$$

where the (low frequency) elastic modulus

$$
E=\left(A_{1} / A_{0}\right) K \text {. }
$$

For human joint, $E \approx 1 \mathrm{MPa}$.

The average interfacial separation $s$ satisfies ${ }^{25,26,28,29}$

$$
\frac{d s}{d t}=-\frac{s^{3} \phi_{\mathrm{p}}(s)}{\eta w^{2}} p_{\text {fluid }}(t)+J_{\perp}(t) .
$$

If $p_{0}$ is the nominal contact pressure then

$$
p_{\text {fluid }}=p_{0}-p_{\text {solid }} \text {. }
$$

Here we will use the asymptotic relation ${ }^{35}$

$$
p_{\text {solid }}=\beta E^{*} \exp \left(-\frac{s}{s_{0}}\right),
$$

where $s_{0}=h_{\text {rms }} / \alpha$, where $\alpha \approx 0.5$ and where $h_{\text {rms }}$ is the rootmean-square roughness. Let us measure the pressure in units of $p_{0}$, separation in units of $h_{\mathrm{rms}}$ and time in units of

$$
\tau=\frac{\eta w^{2} s_{0}}{h_{\mathrm{rms}}^{3} p_{0}}=\frac{\eta w^{2}}{\alpha h_{\mathrm{rms}}^{2} p_{0}} .
$$

We get

$$
\frac{d s}{d t}=-\alpha^{-1} \phi_{\mathrm{p}}(s) s^{3}\left(1-p_{\text {solid }}\right)+\frac{L}{h_{\mathrm{rms}}} \frac{p_{0}}{E} \frac{d p_{\text {solid }}}{d t} .
$$

Using (25), we can also write this as

$$
\frac{d s}{d t}=-\frac{\alpha^{-1} \phi_{\mathrm{p}}(s) s^{3}\left(1-p_{\text {solid }}\right)}{1+\alpha\left(L / h_{\mathrm{rms}}\right)\left(p_{0} / E\right) p_{\text {solid }}} .
$$




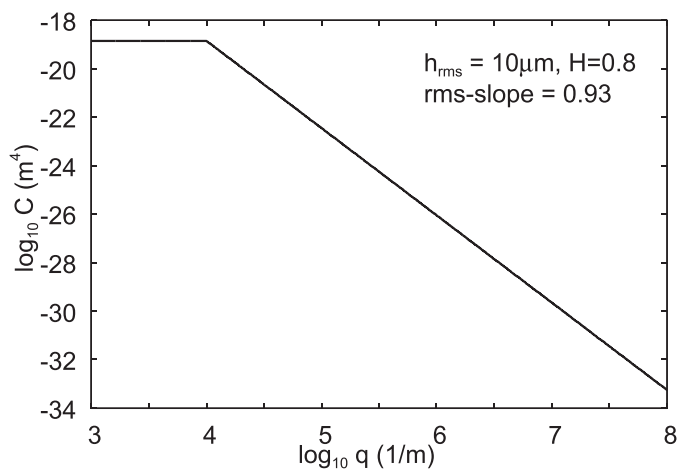

FIG. 5. The surface roughness power spectrum $C(q)$ as a function of the wavenumber $q$ (log-log scale).

Let us now present some numerical results. We consider the contact between a rigid rectangular block of thickness $L=0.2 \mathrm{~cm}$, with a flat surface and with the width $w=2 \mathrm{~cm}$ in the fluid flow direction, squeezed against an elastic or poroelastic solid with a randomly rough surface with the surface roughness power spectrum $C(q)$ shown in Fig. 5. The surface is self affine fractal with the root-mean-square roughness amplitude $10 \mu \mathrm{m}$ and the fractal dimension $D_{f}=3-H=2.2$. The fluid flow at the interface is studied using the Reynolds equation with the additional source term (21). In the fluid flow equation, enter the fluid pressure flow factor $\phi_{\mathrm{p}}$, which depends on $C(q)$, which we calculate using the Bruggeman effective medium theory combined with the Persson contact mechanics theory, as described in earlier publications. ${ }^{28}$ In Fig. 6, we show the pressure flow factor $\phi_{\mathrm{p}}$ as a function of the average interfacial separation $u$ (in units of the rms-roughness amplitude $\left.h_{\mathrm{rms}}=10 \mu \mathrm{m}\right)$.

Let us now consider the fluid squeeze-out. At time $t=0$, we assume the average surface separation $u=10^{-4} \mathrm{~m}$. Fig. 7 shows the average interfacial separation as a function of time (log-log scale). We have assumed that a rigid rectangular strip (width $w=2 \mathrm{~cm}$ ) is squeezed in a fluid (viscosity $\eta=1 \mathrm{~Pa} \mathrm{~s}$ ) against a solid with the surface roughness power spectrum shown in Fig. 5. The squeezing pressure is applied at $t=0$ and increases linearly with time during the first $0.01 \mathrm{~s}$ to its final value $p=1 \mathrm{MPa}$. The red and green lines are for an elastic solid with the effective elastic modulus $E=1 \mathrm{MPa}$, and the pink lines are for a poroelastic solid where the elastic network

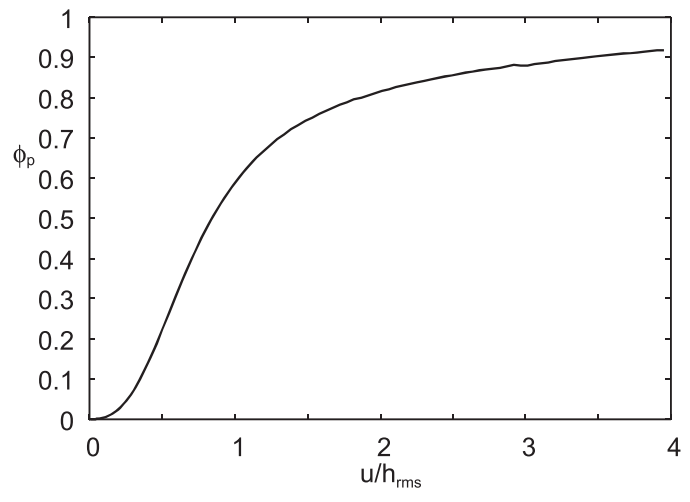

FIG. 6. The pressure flow factor $\phi_{\mathrm{p}}$ as a function of the average interfacial separation $u$ (in units of the rms-roughness amplitude $h_{\mathrm{rms}}=10 \mu \mathrm{m}$ ).

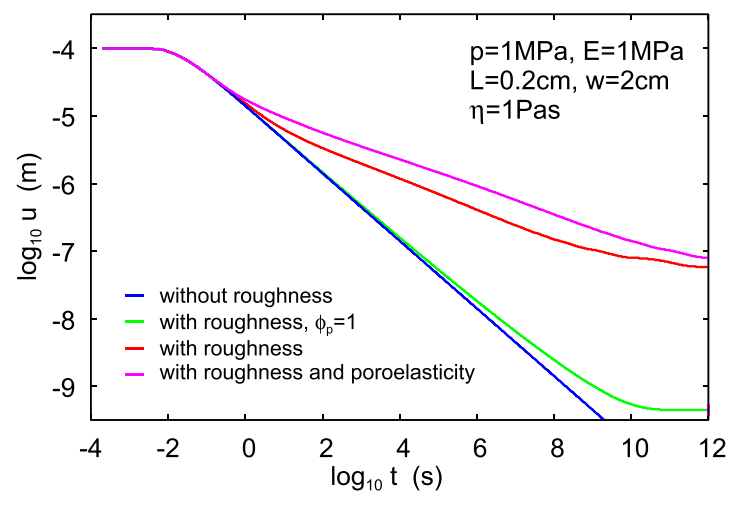

FIG. 7. The average interfacial separation as a function of time (log-log scale). For a rigid rectangular strip (width $w=2 \mathrm{~cm}$ ) squeezed in a fluid (viscosity $\eta=1 \mathrm{~Pa} \mathrm{~s}$ ) against a solid with the surface roughness power spectrum shown in Fig. 5. The squeezing pressure is applied at $t=0$ and increases linearly with time during the first $0.01 \mathrm{~s}$ to its final value $p=1 \mathrm{MPa}$. The red and green lines are for an elastic solid with the elastic modulus $E=1 \mathrm{MPa}$, and the pink line is for a poroelastic solid where the elastic network has the modulus $E=1 \mathrm{MPa}$. The green line is obtained with the pressure flow factor $\phi_{\mathrm{p}}=1$, while the red and pink lines are with the pressure flow factor shown in Fig. 6. The blue line is for rigid solids with flat surfaces (no surface roughness).

has the modulus $E=1 \mathrm{MPa}$. The green line is obtained with the pressure flow factor $\phi_{\mathrm{p}}=1$, while the red and pink lines are with the pressure flow factor shown in Fig. 6. The blue line is for a rigid and flat substrate.
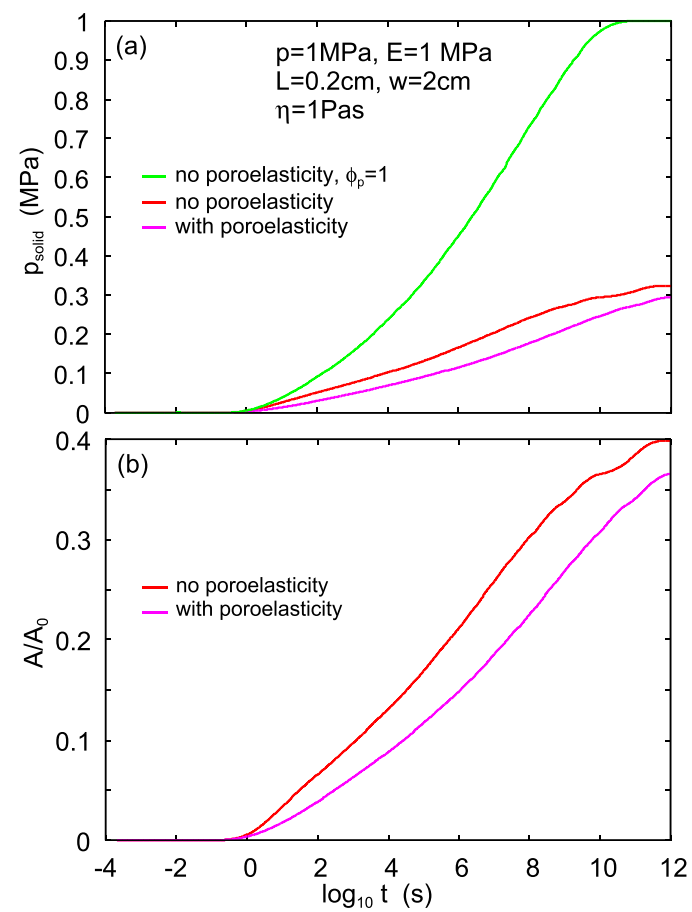

FIG. 8. The (a) solid contact pressure $p_{\text {solid }}$ and (b) the area of contact $A$ (in units of the nominal contact area $A_{0}$ ), as a function of the logarithm of time $t$. For a rigid rectangular strip (width $w=2 \mathrm{~cm}$ ) squeezed in a fluid (viscosity $\eta=1 \mathrm{~Pa}$ s) against a solid with the surface roughness power spectrum shown in Fig. 5. The squeezing pressure is applied at $t=0$ and increases linearly with time during the first $0.01 \mathrm{~s}$ to its final value $p=1 \mathrm{MPa}$. The red and green lines are for an elastic solid with the elastic modulus $E=1 \mathrm{MPa}$, and the pink lines are for a poroelastic solid where the elastic network has the modulus $E=1 \mathrm{MPa}$. The green line is obtained with the pressure flow factor $\phi_{\mathrm{p}}=1$, while the red and pink lines are with the pressure flow factor shown in Fig. 6. 


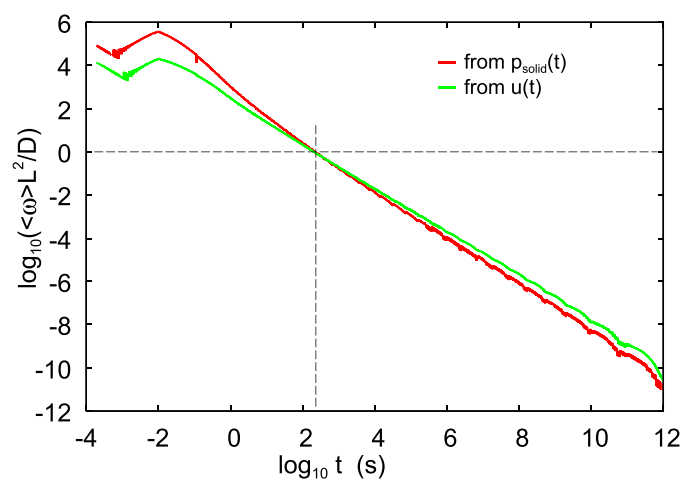

FIG. 9. The quantity $\langle\omega\rangle L^{2} / D$ as a function of time (log-log scale), where the effective frequency $\langle\omega\rangle$ is calculated from the time variation of $p_{\text {cont }}(t)$ (red line) or $u(t)$ (green line) using $\langle\omega\rangle=p^{\prime}{ }_{\text {cont }}(t) / p_{\text {cont }}(t)$ and $u^{\prime}(t) / u(t)$, respectively.

Fig. 8 shows (a) the solid contact pressure $p_{\text {solid }}$ and (b) the area of contact $A$ (in units of the nominal contact area $A_{0}$ ), as a function of the logarithm of time $t$. The red and green lines are again for an elastic solid with the elastic modulus $E=1 \mathrm{MPa}$, and the pink lines are for a poroelastic solid where the elastic network has the modulus $E=1 \mathrm{MPa}$. The green line is obtained with the pressure flow factor $\phi_{\mathrm{p}}=1$, while the red and pink lines are with the pressure flow factor shown in Fig. 6.

For the human cartilage, experiments have shown that the diffusivity $D \approx 4 \times 10^{-9} \mathrm{~m}^{2} / \mathrm{s}$. Thus we get the characteristic time (relaxation time) $\tau_{0}=L^{2} / D \approx 1000 \mathrm{~s}$. Because of the assumption (21), the numerical results presented above are only valid for times $t>\tau_{0}$, i.e., for $t$ larger than about $\approx 10^{3} \mathrm{~s}$. Thus, only when the time variation of $p_{\text {cont }}(t)$ or $u(t)$ becomes slower than $\tau_{0}$, the approximation (21) holds. As shown in Fig. 9, this is obeyed when $t>\tau_{0}$.

\section{DISCUSSION}

The study above shows that the fluid squeeze-out occurs over a very long time period. This is partly due to the large width of the contact region $(w=2 \mathrm{~cm})$ but mainly due to trapped islands of pressurized fluid which carry a part of the external load. Trapped islands of the fluid can occur at any stage in the squeeze-out but when the (relative) contact area $A / A_{0} \approx 0.42$ the contact area percolates, ${ }^{22}$ and at this point in time most of the liquid at the interface will be trapped.

Fig. 8 shows that some asperity contact occurs already after a short contact time, e.g., after $10 \mathrm{~min}$ the contact area $A / A_{0} \approx 0.1$ for the elastic solid and $\approx 0.07$ for the poroelastc solid, although the latter result is inaccurate because longer contact time is necessary for the asymptotic result (21) to hold accurately. Anyway, the numerical results indicate that the area of contact is only weakly affected by poroelasticity and suggest similar break-loose friction force in both cases, assuming that the break-loose friction force is proportional to the area of contact.

Fig. 8 shows that the (average) pressure in the asperity contact regions, given by $p_{\text {solid }} A_{0} / A$, is only of order $1 \mathrm{MPa}$. The cartilage consists of polar molecules with charged groups and counter-ions. For such systems, experiments have shown for contact pressures up to $\sim 1 \mathrm{MPa}$ the osmotic pressure, and for higher contact pressures (up to $\sim 10 \mathrm{MPa}$, or more) hydration lubrication, ${ }^{30,31}$ (the hypothesis that hydration shells surrounding charges act as lubrication elements in boundary layers) could be important and result in extremely low friction. Thus, recent studies suggest that the very low friction observed for the synovial joint, for contact pressures up to $\sim 10 \mathrm{MPa}$, result from highly hydrated phosphocholine groups via the hydration lubrication mechanism. ${ }^{31,32}$

Here I would like to suggest another way that the poroelasticity could reduce friction and wear. It has recently been found that elastohydrodynamic effects can have a huge effect on the sliding friction for soft elastic solids. Thus, in Ref. 33, it was observed that when soft rubber blocks are slid with a constant driving force on a lubricated substrate, the motion stops within a few seconds and, if the driving force is big enough, the lubricant is completely removed from the interface. For a block made from an elastically hard solid like Poly(methyl methacrylate) (PMMA or plexiglass), we instead observed that

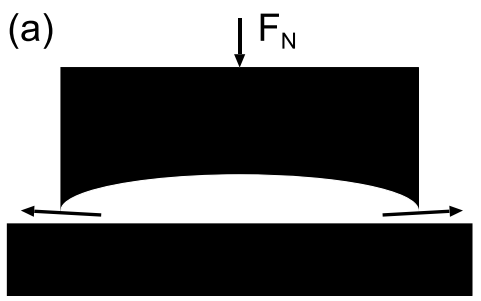

squeezing

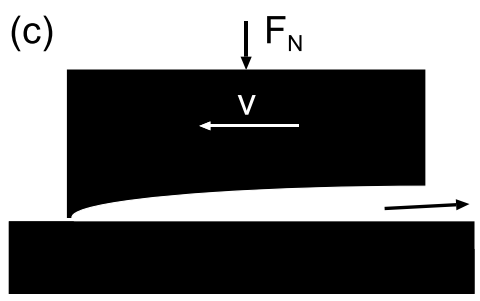

sliding

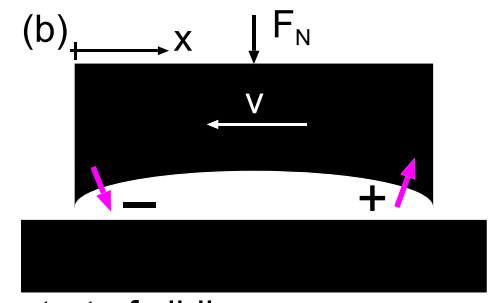

start of sliding

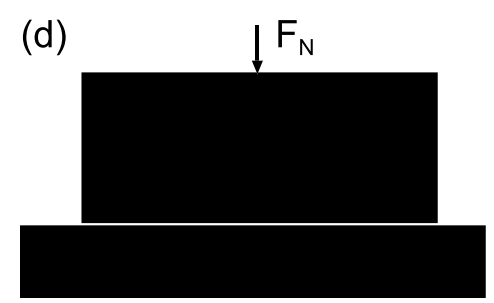

end of sliding
FIG. 10. During squeezing the bottom surface of the rubber block deform as in (a). During sliding the surface separation changes as indicated in (b)-(d). Reproduced with permission from J. Chem. Phys. 144, 124903 (2016). Copyright 2016 AIP Publishing LLC. 
the speed of the block (and the fluid film thickness) increased with time.

In Ref. 33, we proposed the following explanation for the observations. When a rigid body with a flat surface is squeezed against a flat rigid countersurface in a fluid, because of the fluid viscosity a pressure will develop in the fluid, which is maximal in the center of the contact region (see, e.g., Refs. 1 and 2). For elastic solids, the fluid pressure will deform the solid such that the separation between the solids will have a maximum at the center of the contact region, see Fig. 10(a). These deformations of the bottom surface of the block increases when the elastic modulus of the solids decreases. With the nominal contact pressures used in Ref. 33, the effect is large for the rubber materials but negligible for the PMMA.

If we now start to slide, the fluid pressure will decrease at the inlet side because of the increasing surface separation with increasing $x$, while it will increase on the exit side due to the decreasing surface separation with increasing $x$. Thus, on the rubber surface will act a fluid pressure which will deform the rubber as indicated by the pink arrows in (b). Hence, during sliding the surfaces will be deformed as in (c), which will strongly reduce the inflow of the fluid at the inlet side and make it easy for the fluid to disappear on the exit side. As a result, the separation between the surfaces will rapidly decrease, and at the stop of sliding, the surface separation may be as in (d). This explains why for the silicon rubber block, sliding just $\sim 3$ times the width of the block in the sliding direction results in a nearly dry contact area. We refer to the process above as the dynamic scrape mechanism.

For a poroelastic solid, the dynamical scape process (and cavitation in negative pressure regions, which could have a detrimental influence on the wear) will be suppressed for the reason illustrated in Fig. 11. Thus, the negative fluid pressure at the leading edge of the confined fluid region will result in a flow of fluid from the poroelastic solid into the space between the solids, which will reduce the tendency to close the gap at the front edge of the confined fluid region. This will reduce the friction and wear, and we propose that this may be the most important reason for why human and animal cartilages are porous, fluid filled, structures.

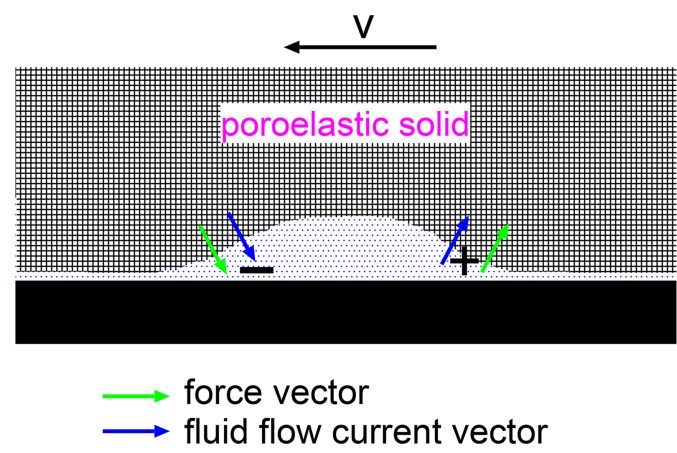

FIG. 11. For a poroelastic solid, the negative fluid pressure at the leading edge of the confined fluid will result in a flow of fluid from the poroelastic solid into the space between the solids, which will reduce the tendency for the gap between the solids to close the front edge of contact. This will reduce the friction and wear

\section{SUMMARY AND CONCLUSION}

In Refs. 13 and 14, Mow and coworkers reported on fundamental work for some simple poroelastic contact mechanics configurations, such as fluid squeeze-out through a rigid filter or a moving parabolic load. The present contribution consider the influence of the surface roughness on many length scales on the fluid flow at the interface between a poroelastic solid and a rigid randomly rough countersurface. This is a complex topic and is studied for the first time in this paper using an accurate treatment of the interfacial fluid flow.

I have presented a simple model of a fluid filled poroelastic solid and considered the contact mechanics (fluid squeeze-out) between a poroelastic solid and a hard solid with a randomly rough surface. I have shown that for the poroelastic solid the fluid squeeze-out from the interface is slower than for the corresponding elastic solid, but the effect is not so large, and is unlikely to be the fundamental reason for the low friction of the human joint. I have also discussed the role of poroelasticity on the break-loose friction force and suggested a mechanism by which the break-lose friction force is reduced for poroelastic solids. The low friction observed for the human joints may result from osmotic pressure and, as recently proposed, from hydration lubrication. ${ }^{31,32}$

The human cartilage is more complex than the very simple model used above. Thus, for example, it has properties which depend on the distance $z$ from the surface. The contact mechanics model we used can also be applied to such layered materials. ${ }^{34}$ Another complication result from the fact that the cartilage is made from molecules which are charged when surrounded by water. This result in osmotic pressure effects as the water is squeezed out from the material. However, more severe is the assumption made above about the fluid flow (we only allowed for the fluid flow in the $z$-direction) and the consideration of the long-time response of the poroelastic solid. In a future publication, I hope to present a more general study where both these constrains are removed.

\section{ACKNOWLEDGMENTS}

This work was performed within a Reinhart-Koselleck project funded by the Deutsche Forschungsgemeinschaft (DFG). We would like to thank DFG for the project support under the reference German Research Foundation DFG-Grant No. MU 1225/36-1. The research work was also supported by the DFG-Grant No. PE 807/10-1. This work is supported in part by COST Action No. MP1303.

\section{APPENDIX A: CONNECTION TO THE STANDARD MODEL OF POROELASTIC SOLIDS}

The standard equations for the mechanical properties of the fluid filled poroelastic solid, with isotropic properties, can be easily obtained from the equations derived in Sec. II A. Thus for an isotropic elastic solid symmetry arguments demand that (6) must take the form

$$
-\nabla p+K_{1} \nabla \nabla \cdot \mathbf{u}+K_{2} \nabla^{2} \mathbf{u}=0
$$


and (5) takes the form

$$
c \frac{\partial \mathbf{u}}{\partial t}=\nabla p
$$

Since in the present case the fluid velocity vanishes, we can also write this equation as

$$
c\left(\mathbf{v}_{\text {solid }}-\mathbf{v}_{\text {fluid }}\right)=\nabla p .
$$

This equation can be interpreted as follows: the driving force for fluid flow, the $\nabla p$-term, is balanced by a frictional drag force between the fluid and the solid (the left hand side in (A2)), which depends on the relative velocity of the two components. Note, however, that the microscopic origin of the friction in the model studied above is not (mainly) the friction against the solid walls inside the cavities, but rather due to the energy dissipation, due to the viscosity of the fluid, as it is squeezed through the narrow pore channels.

The coefficient $c$ in (A2) can be measured in a simple fluid-flow experiment: a rectangular disk (thickness $H$ ) of the poroelastic material is surrounded by the fluid on the upper and lower side with the pressures $p_{\mathrm{a}}$ and $p_{\mathrm{b}}$, respectively. This will result in fluid flow (leakage) from the high pressure side to the low pressure side and (10) takes the form $c v_{\text {fluid }}=\left(p_{\mathrm{a}}-p_{\mathrm{b}}\right) / H$ (where we assumed $p_{\mathrm{a}}>p_{\mathrm{b}}$ ), and by measuring the flow velocity $v_{\text {fluid }}$, one can determine $c$ which is usually denoted as the coefficient of the diffusive resistance. Note, however, that $v_{\text {fluid }}$ is the (average) fluid flow inside the material which differ from the fluid flow velocity $v_{\text {fluid }}^{*}$ measured outside the poroelastic solid: volume conservation requires that $v_{\text {fluid }}^{*} A_{0}=v_{\text {fluid }} A_{1}$, where $A_{0}$ is the sample cross section area orthogonal to the fluid flow direction and $A_{1} / A$ the fraction of the cross section area $A_{0}$ which is occupied by the fluid. The ratio $A_{1} / A_{0}$ can be determined by studying a cross section area of the material.

In addition to the equations above, if the fluid and solid material can be considered as incompressible, the equation of continuity takes the form

$$
\nabla \cdot\left(\alpha_{\text {solid }} \mathbf{v}_{\text {solid }}+\alpha_{\text {fluid }} \mathbf{v}_{\text {fluid }}\right)=0
$$

where $\alpha_{\text {solid }}$ and $\alpha_{\text {fluid }}$ are the fraction of the material occupied by the solid and fluid components, respectively.

\section{APPENDIX B: FLUID FLOW CURRENT $J_{\perp}(t)$}

From (17), we get

$$
J_{\perp}(t)=-\kappa \frac{B}{A_{1}} \int d \omega e^{-i \omega t} p_{\text {solid }}(\omega) \gamma(\omega) \frac{1-e^{\gamma(\omega) 2 L}}{1+e^{\gamma(\omega) 2 L}} .
$$

Substituting

$$
p_{\text {solid }}(\omega)=\frac{1}{2 \pi} \int d t^{\prime} p_{\text {solid }}\left(t^{\prime}\right) e^{i \omega t^{\prime}}
$$

in (B1) gives

$$
J_{\perp}(t)=-\kappa \frac{B}{A_{1}} \int d t^{\prime} G\left(t-t^{\prime}\right) p_{\text {solid }}\left(t^{\prime}\right),
$$

where

$$
G(t)=\frac{1}{2 \pi} \int d \omega e^{-i \omega t} \gamma(\omega) \frac{1-e^{\gamma(\omega) 2 L}}{1+e^{\gamma(\omega) 2 L}},
$$

where

$$
\gamma=\left(-\frac{i \omega}{D}\right)^{1 / 2}
$$

The integral (B3) can be performed using the complex integration. Note that

$$
f(\omega)=1+e^{\gamma(\omega) 2 L}=0,
$$

if

$$
\gamma(\omega) 2 L=i \pi(1+2 n),
$$

where $n$ is an integer. Thus the solution to (B4) is $\omega=\omega_{n}$, where

$$
\omega_{n}=-i D\left(\frac{\pi}{2 L}\right)^{2}(1+2 n)^{2}=-i \alpha_{n},
$$

where $n=0,1, \ldots$ Note that $\operatorname{Im} \omega_{n}<0$, so all the poles $\omega=\omega_{n}$ occur in the lower $\omega$ half plane. It follows that for $t<0$ the function $G(t)=0$. When $t>0$, we close the integral (B3) in the lower $\omega$ half plane. It is easy to show that there is no contribution to the integral from the semi-circle at infinite $|\omega|$. Thus the integral will be equal to the sum over the poles $\omega=$ $\omega_{n}$, and expanding the denominator $f(\omega)=f^{\prime}\left(\omega_{n}\right)\left(\omega-\omega_{n}\right)$, we get

$$
\begin{aligned}
G(t) & =\frac{1}{2 \pi} \int d \omega e^{-i \omega t} \sum_{n} \frac{2 \gamma\left(\omega_{n}\right)}{f^{\prime}\left(\omega_{n}\right)\left(\omega-\omega_{n}\right)} \\
& =\theta(t) \sum_{n} e^{-\alpha_{n} t} \frac{-2 i \gamma\left(\omega_{n}\right)}{f^{\prime}\left(\omega_{n}\right)},
\end{aligned}
$$

where $\theta(t)=1$ for $t>0$ and $=0$ for $t<0$. Next using that

$$
f^{\prime}\left(\omega_{n}\right)=-\gamma^{\prime}\left(\omega_{n}\right) 2 L=-\gamma\left(\omega_{n}\right)\left(L / \omega_{n}\right)
$$

we get from (B6) and (B7),

$$
\begin{aligned}
G(t) & =\theta(t) \sum_{n} e^{-\alpha_{n} t} \frac{-2 i \gamma\left(\omega_{n}\right)}{-\gamma\left(\omega_{n}\right)\left(L / \omega_{n}\right)} \\
& =\frac{\pi^{2} D}{2 L^{3}} \theta(t) \sum_{n} e^{-\alpha_{n} t}(1+2 n)^{2}
\end{aligned}
$$

Thus we can write

$$
J_{\perp}(t)=-\kappa \frac{B}{A_{1}} \frac{\pi^{2} D}{2 L^{3}} \int_{-\infty}^{t} d t^{\prime} H\left(t-t^{\prime}\right) p_{\text {solid }}\left(t^{\prime}\right),
$$

where

$$
H(t)=\sum_{n} e^{-\alpha_{n} t}(1+2 n)^{2}
$$

Note that in most applications, one must assume that the contact pressure is turned on at some time which we denote as $t=0$. In this case $p_{\text {solid }}=0$ for $t<0$, so that

$$
J_{\perp}(t)=-\kappa \frac{B}{A_{1}} \frac{\pi^{2} D}{2 L^{3}} \int_{0}^{t} d t^{\prime} H\left(t-t^{\prime}\right) p_{\text {solid }}\left(t^{\prime}\right) .
$$

For long times, $p_{\text {solid }}(t)$ varies slowly with time compared to the function $H(t)$, and we can write

$$
p_{\text {solid }}\left(t^{\prime}\right)=p_{\text {solid }}(t)+p_{\text {solid }}^{\prime}(t)\left(t^{\prime}-t\right)+\cdots .
$$

We get

$$
J_{\perp}(t)=\kappa \frac{B}{A_{1}} \frac{\pi^{2} D}{2 L^{3}}\left[H_{0} p_{\text {solid }}(t)+H_{1} p_{\text {solid }}^{\prime}(t)+\cdots\right],
$$

where

$$
H_{0}=-\int_{0}^{t} d t^{\prime} H\left(t-t^{\prime}\right)=-\int_{0}^{t} d t H(t),
$$

$$
H_{1}=\int_{0}^{t} d t^{\prime} H\left(t-t^{\prime}\right)\left(t-t^{\prime}\right)=\int_{0}^{t} d t H(t) t .
$$


Note that

$$
\begin{aligned}
H_{1} & =\sum_{n}\left(1-e^{-\alpha_{n} t}\right) \frac{(1+2 n)^{2}}{\alpha_{n}^{2}} \\
& =\left(\frac{2 L}{\pi}\right)^{4} \frac{1}{D^{2}} \sum_{n}\left(1-e^{-\alpha_{n} t}\right) \frac{1}{(1+2 n)^{2}} .
\end{aligned}
$$

Here we are interested in such long times that $D t / L^{2}>>1$ in which case the $\exp \left(-\alpha_{n} t\right)$ term in (B12) can be neglected. Thus we get

$$
J_{\perp}(t)=\kappa \frac{B}{A_{1}} \frac{8 L}{D \pi^{2}} \sum_{n} \frac{1}{(1+2 n)^{2}} p_{\text {solid }}^{\prime}(t)+\cdots .
$$

Using that $D=K / c=K \kappa B / A_{1}$ gives

$$
J_{\perp}(t)=\frac{8 L}{\pi^{2} K} \sum_{n} \frac{1}{(1+2 n)^{2}} p_{\text {solid }}^{\prime}(t)+\cdots
$$

Next using that

$$
\sum_{n} \frac{1}{(1+2 n)^{2}}=\frac{\pi^{2}}{8}
$$

we get to leading order for long time

$$
J_{\perp}(t)=\frac{L}{K} p_{\text {solid }}^{\prime}(t),
$$

which agrees with (21). Note that the derivation of this result is much simpler in the frequency-space than in the time-space.

\section{APPENDIX C: ELASTIC SURFACE DEFORMATION $u(t)$}

The elastic deformation field $u(z, \omega)$ is given by (15),

$$
u(z, \omega)=-\frac{A_{0}}{A_{1}} \frac{p_{\text {solid }}}{K \gamma} \frac{e^{\gamma z}-e^{\gamma(2 L-z)}}{1+e^{\gamma 2 L}} .
$$

The surface deformation $u(\omega)=u(0, \omega)$ is given by

$$
u(\omega)=-\frac{A_{0}}{A_{1}} \frac{p_{\text {solid }}}{K \gamma} \frac{1-e^{\gamma 2 L}}{1+e^{\gamma 2 L}} .
$$

Let us first consider the case when $p_{\text {solid }}(t)=0$ for $t<0$ and $p_{\text {solid }}(t)=p_{\text {solid }}^{0}$ constant for $t>0$. In this case,

$$
p_{\text {solid }}(\omega)=\frac{p_{\text {solid }}^{0}}{2 \pi(\epsilon-i \omega)}
$$

where $\epsilon=0^{+}$is an infinitesimal small positive number. Thus we get

$$
\begin{aligned}
u(t) & =\int d \omega u(\omega) e^{-i \omega t} \\
& =\frac{A_{0}}{A_{1}} \frac{p_{\text {solid }}^{0}}{K} \frac{1}{2 \pi i} \int d \omega \frac{1}{(\omega+i \epsilon) \gamma(\omega)} \frac{1-e^{\gamma(\omega) 2 L}}{1+e^{\gamma(\omega) 2 L}} e^{-i \omega t}
\end{aligned}
$$

Consider now the integral

$$
I=\frac{1}{2 \pi i} \int d \omega \frac{1}{(\omega+i \epsilon) \gamma(\omega)} \frac{1-e^{\gamma(\omega) 2 L}}{1+e^{\gamma(\omega) 2 L}} e^{-i \omega t} .
$$

The integrand has poles in the lower $\omega$-half planes at $\omega=-i \epsilon$ and at $\omega=\omega_{n}=-i \alpha_{n}$ (see Appendix B). Since

$$
\frac{1}{\gamma(\omega)} \frac{1-e^{\gamma(\omega) 2 L}}{1+e^{\gamma(\omega) 2 L}} \rightarrow-L,
$$

as $\omega \rightarrow 0$, the contribution to the integral $I$ from the pole $\omega=-i \epsilon$ will be just $L$. The contribution from the poles $\omega=\omega_{n}=-i \alpha_{n}$ can be calculated just as in Appendix B. Thus we get

$$
I=L-\sum_{n} \frac{2 D}{L \alpha_{n}} e^{-\alpha_{n} t}=L\left(1-\sum_{n} \frac{2 D}{L^{2} \alpha_{n}} e^{-\alpha_{n} t}\right)
$$

or

$$
I=L\left(1-\frac{8}{\pi^{2}} \sum_{n} \frac{1}{(1+2 n)^{2}} e^{-\alpha_{n} t}\right)
$$

Thus we get

$$
u(t)=L \frac{A_{0}}{A_{1}} \frac{p_{\text {solid }}^{0}}{K}\left(1-\frac{8}{\pi^{2}} \sum_{n} \frac{1}{(1+2 n)^{2}} e^{-\alpha_{n} t}\right),
$$

a result already obtained by Mow and coworkers, ${ }^{13,14}$ and found to be in good agreement with confined compression data for the human cartilage. Here a free-draining, rigid filter, was squeezed against a cylindrical plug of the cartilage in a rigid confining chamber. The load or squeezing force was constant and the vertical displacement of the filter measured as a function of the squeezing time.

Let us now consider the general case where $p_{\text {solid }}(t)$ depends on time in an arbitrary way. Let us write

$$
u(\omega)=F(\omega) p_{\text {solid }}(\omega),
$$

where

$$
F(\omega)=-\frac{A_{0}}{A_{1}} \frac{1}{K \gamma} \frac{1-e^{\gamma 2 L}}{1+e^{\gamma 2 L}}
$$

Thus we get

$$
\begin{aligned}
u(t) & =\int d \omega F(\omega) p_{\text {solid }}(\omega) e^{-i \omega t} \\
& =\int d t^{\prime} p_{\text {solid }}\left(t^{\prime}\right) \frac{1}{2 \pi} \int d \omega F(\omega) e^{-i \omega\left(t-t^{\prime}\right)} .
\end{aligned}
$$

Now consider the integral

$$
J=-\frac{1}{2 \pi} \int d \omega \frac{1}{\gamma(\omega)} \frac{1-e^{\gamma(\omega) 2 L}}{1+e^{\gamma(\omega) 2 L}} e^{-i \omega t} .
$$

Using the results of Appendix B, we obtain

$$
J=\frac{2 D}{L} \theta(t) \sum_{n} e^{-\alpha_{n} t},
$$

where $\theta(t)=1$ for $t>0$ and $=0$ for $t<0$. Combining this with (C2) gives

$$
u(t)=\frac{A_{0}}{A_{1}} \frac{2 D}{L} \int_{-\infty}^{t} d t^{\prime} p_{\text {solid }}\left(t^{\prime}\right) \sum_{n} e^{-\alpha_{n}\left(t-t^{\prime}\right)} .
$$

If $p_{\text {solid }}(t)=0$ for $t<0$ and a constant $p_{\text {solid }}^{0}$ for $t>0$, we get

$$
u(t)=L \frac{A_{0}}{A_{1}} \frac{p_{\text {solid }}^{0}}{K} \frac{8}{\pi^{2}} \sum_{n} \frac{1}{(2 n+1)^{2}}\left(1-e^{-\alpha_{n} t}\right) .
$$

Using (B13), this gives

$$
u(t)=L \frac{A_{0}}{A_{1}} \frac{p_{\text {solid }}^{0}}{K}\left(1-\frac{8}{\pi^{2}} \sum_{n} \frac{1}{(2 n+1)^{2}} e^{-\alpha_{n} t}\right),
$$

which agrees with (C1). 
${ }^{1}$ B. N. J. Persson, Sliding Friction: Physical Principles and Applications (Springer, Heidelberg, 2000).

${ }^{2}$ E. Gnecco and E. Meyer, Elements of Friction Theory and Nanotribology (Cambridge University Press, 2015).

${ }^{3}$ A. Vanossi, N. Manini, M. Urbakh, S. Zapperi, and E. Tosatti, Rev. Mod. Phys. 85, 529 (2013).

${ }^{4}$ J. Krim, Sci. Am. 275, 74 (1996).

${ }^{5}$ P. S. Walker, D. Dowson, M. D. Longfield, and V. Wright, Ann. Rheum. Dis. 27, 512 (1968).

${ }^{6}$ S. Lee and N. D. Spencer, Science 319, 575 (2008).

${ }^{7}$ J. Ahmed, H. Guo, T. Yamamoto, T. Kurokawa, M. Takahata, T. Nakajima, and J. P. Gong, Macromolecules 47, 3101 (2014).

${ }^{8}$ A. C. Dunn, W. G. Sawyer, and T. E. Angelini, Tribol. Lett. 54, 59 (2014).

${ }^{9}$ S. Beer and M. H. Müser, Macromolecules 47, 7666 (2014).

${ }^{10}$ T. Baumberger, C. Caroli, and D. Martina, Eur. Phys. J. E 21, 81 (2006).

${ }^{11}$ C. Creton and M. Ciccotti, Rep. Prog. Phys. 79, 046601 (2016).

${ }^{12}$ J. B. Sokoloff, Phys. Rev. E 90, 032408 (2014).

${ }^{13}$ V. C. Mow, S. C. Kuei, W. M. Lai, and C. G. Armstrong, J. Biomech. Eng. 102, 73 (1980).

${ }^{14}$ M. K. Kwan, W. M. Lai, and V. C. Mow, Ann. Biomed. Eng. 12, 537 (1984).

${ }^{15}$ P. Goodwyn, A. Peressadko, H. Schwarz, V. Kastner, and S. Gorb, J. Comp. Physiol., A 192, 1233 (2006).

${ }^{16}$ W. Federle, W. J. P. Barnes, P. D. W. Baumgartner, and J. M. Smith, J. R. Soc., Interface 3, 689 (2006).

${ }^{17}$ B. N. J. Persson, J. Phys.: Condens. Matter 22, 265004 (2010).

${ }^{18}$ B. N. J. Persson, J. Chem. Phys. 115, 3840 (2001).
${ }^{19}$ A. Almqvist, C. Campana, N. Prodanov, and B. N. J. Persson, J. Mech. Phys. Solids 59, 2355 (2011).

${ }^{20}$ B. Lorenz and B. N. J. Persson, Eur. Phys. J. E 31, 159 (2010).

${ }^{21}$ B. Lorenz and B. N. J. Persson, EPL 86, 44006 (2009).

${ }^{22}$ W. B. Dapp, A. Lücke, B. N. J. Persson, and M. H. Müser, Phys. Rev. Lett. 108, 244301 (2012).

${ }^{23}$ D. A. G. Bruggeman, Ann. Phys. 24, 636 (1935).

${ }^{24} \mathrm{O}$. Reynolds, "On the theory of lubrication and its application to Mr. Beauchamp Tower's experiments, including an experimental determination of the viscosity of olive oil," Philos. Trans. R. Soc. London 177, 157 (1886).

${ }^{25}$ B. Lorenz and B. N. J. Persson, J. Phys.: Condens. Matter 23, 355005 (2011).

${ }^{26}$ B. Lorenz and B. N. J. Persson, Eur. Phys. J. E 32, 281 (2010).

${ }^{27}$ L. D. Landau and E. M. Lifshitz, Fluid Mechanics (Pegamon Press, 1959), Sec. 17 (Eq. 17.10),

${ }^{28}$ B. N. J. Persson and M. Scaraggi, Eur. Phys. J. E 34, 1 (2011).

${ }^{29}$ M. Scaraggi and B. N. J. Persson, Tribol. Lett. 47, 409 (2012).

${ }^{30}$ N. Dhopatkar, A. P. Defante, and A. Dhinojwala, Sci. Adv. 2, e1600763 (2016).

${ }^{31}$ S. Jahn and J. Klein, Macromolecules 48, 5059 (2015).

${ }^{32}$ J. Seror, L. Zhu, R. Goldberg, A. J. Day, and J. Klein, Nat. Commun. 6, 6497 (2015).

${ }^{33}$ W. Hutt and B. N. J. Persson, J. Chem. Phys. 144, 124903 (2016).

${ }^{34}$ B. N. J. Persson, J. Phys.: Condens. Matter 24, 095008 (2012).

${ }^{35}$ B. N. J. Persson, Phys. Rev. Lett. 99, 125502 (2007). 